\title{
Produção de amora-preta e amora-vermelha em Lavras - MG
}

\author{
Production of blackberry and redberry in Lavras - MG, Brazil
}

\author{
Paula Nogueira Curi ${ }^{I}$ Rafael Pio ${ }^{I^{*}}$ Pedro Henrique Abreu Moura ${ }^{\mathrm{I}}$ Maraísa Hellen Tadeu \\ Paulyene Vieira Nogueira ${ }^{\mathrm{I}}$ Moacir Pasqual $^{\mathrm{I}}$
}

\section{RESUMO}

O cultivo de amoras está amplamente difundido em zonas temperadas, porém, algumas cultivares são de baixa necessidade em frio e podem ser exploradas em locais com temperaturas mais elevadas. Assim, objetivou-se, com o presente trabalho, avaliar a produção de amoras-pretas e amoravermelha na região de Lavras-MG (clima $\mathrm{Cwb}$ ). O delineamento experimental foi em blocos ao acaso, com quatro blocos $e$ onze tratamentos (cultivares de amoreiras-pretas 'Arapaho', 'Xavante', 'Ébano', 'Comanche', 'Caingangue', 'Choctaw', 'Tupy', 'Guarani', 'Brazos', 'Cherokee' e a amoreira-vermelha, espécie nativa do Brasil). Em cada parcela, foram coletados dados fenológicos e produtivos no ciclo de produção 2010/11 e 2011/12, além da caracterização físico-química dos frutos. As cultivares de amoreira-preta apresentaram ciclo produtivo entre 66 e 133 dias, com colheitas se iniciando em setembro e se estendendo até janeiro; e a amoreira-vermelha com ciclo produtivo de 283 dias. 'Brazos' apresentou a maior produtividade estimada. As cultivares 'Brazos' e 'Guarani' possibilitaram a produção de frutos de maior massa, enquanto que 'Caingangue', 'Tupy' e 'Choctaw' apresentaram bom equilíbrio entre os sólidos solúveis e acidez. A amoreira-vermelha apresentou elevada produção de frutos $(527 \mathrm{~g}$ por planta no $1^{\circ}$ ciclo e $344 \mathrm{~g}$ por planta no $2^{\circ}$ ciclo) e demonstrouse uma excelente opção para o processamento.

Palavras-chave: Rubus spp., fenologia, produtividade, qualidade dos frutos.

\section{ABSTRACT}

Berries are widespread in temperate zones, but some cultivars have low requirements in cold weather and can be exploited in areas with high temperatures. The goal of this study was to evaluate the production of blackberries and redberry in Lavras (climate $C w b$ ). The experimental design was in randomized blocks with four replications and eleven treatments ('Arapaho', 'Xavante', 'Ébano', 'Comanche', 'Caingangue', 'Choctaw',
'Tupy', 'Guarani', 'Brazos' and 'Cherokee' blackberry cultivars and the redberry, native from Brazil). In each experimental plot it was evaluated phonological data, production data during 2010/11 and 2011/12 and physicochemical fruit characteristics. The blackberry cultivars have production cycle between 66 and 133 days, harvest period starting in September until January, and redberry production cycle has 283 days. 'Brazos' presented the highest estimated yield $\left(\mathrm{kg} \mathrm{ha}^{-1}\right)$. 'Brazos' and 'Guarani' cultivars enabled the production of higher fruit mass, while 'Caingangue', 'Tupy' and 'Choctaw' presented a good balance between soluble solids content and titratable acidity. The redberry had a high fruit production $\left(527 \mathrm{~g}\right.$ per plant on $1^{\text {st }}$ cycle and $344 \mathrm{~g}$ per plant on $2^{\text {nd }}$ cycle) and showed to be an excellent choice for processing.

Key words: Rubus spp., phynology, yield, fruits quality.

\section{INTRODUÇ̃̃O}

O interesse pelo consumo da amora-preta e da framboesa (Rubus spp.) aumentou nos últimos anos, devido a esses frutos possuírem quantidades expressivas de compostos fenólicos e carotenoides, que podem auxiliar no combate a doenças degenerativas (GUEDES et al., 2013; SOUZA et al., 2014; CURI et al., 2014). Além desses compostos, podem-se destacar os pigmentos naturais, principalmente a antocianina, que confere uma coloração atraente no processamento desses frutos, especialmente para elaboração de produtos lácteos, geleias e doces em calda (GUEDES et al., 2014; MARO et al., 2014).

No Rio Grande do Sul (clima Cfa, temperado úmido, segundo a classificação climática

IPrograma de Pós-graduação em Agronomia/Fitotecnia, Departamento de Agricultura (DAG), Universidade Federal de Lavras (UFLA), CP 3037, 37200-000, Lavras, MG, Brasil. E-mail: rafaelpio@dag.ufla.br. *Autor para correspondência. 
de Köppen), foi observada uma produtividade média de $15.200 \mathrm{~kg} \mathrm{ha}^{-1}$ com a cultivar de amoreira-preta 'Ébano' (RASEIRA et al., 2007) e 5.169kg ha-1 com a cultivar 'Tupy' (ANTUNES et al., 2010).

Essa fruteira também vem apresentando boa adaptação em clima subtropical, onde algumas cultivares apresentam maiores produtividades em comparação às regiões mais frias e até mesmo com qualidade superior dos frutos (GUEDES et al., 2013). Em Caldas-MG (clima Cwa, subtropical), com a cultivar Brazos, foi obtida uma produtividade de $25.238 \mathrm{~kg} \mathrm{ha}^{-1}$ (ANTUNES et al., 2000), enquanto que, em Santa Helena-PR (clima Cfa, zona subtropical úmida), a cultivar 'Tupy' produziu $6.430 \mathrm{~kg} \mathrm{ha}^{-1}$ (CAMPAGNOLO \& PIO, 2012a).

Como as áreas cultivadas com essa frutífera estão avançando para outras localidades no sudeste brasileiro, a avaliação das cultivares de amoreirapreta em regiões tropicais é de suma importância. Também há necessidade de quantificar a produção e a qualidade dos frutos da amoreira-vermelha $(\boldsymbol{R}$. rosifolius), espécie nativa da serra da Mantiqueira do Brasil (CAMPAGNOLO \& PIO, 2012b).

Assim, o objetivo do presente trabalho foi avaliar a fenologia, desenvolvimento vegetativo, qualidade dos frutos, produtividade e produção de amoreiras-pretas e amoreira-vermelha na região de Lavras-MG.

\section{MATERIAL E MÉTODOS}

O trabalho foi conduzido no município de Lavras-MG, situado a $21^{\circ} 14^{\prime} 06^{\prime \prime}$ de latitude Sul e $45^{\circ} 00^{\prime} 00^{\prime \prime}$ de latitude Oeste, a uma altitude média de 918 metros, no período de novembro de 2009 a janeiro de 2012. O clima da região é do tipo Cwb, (clima mesotérmico ou tropical de altitude), com inverno seco e verão chuvoso, segundo a classificação de Köppen.

Mudas das cultivares de amoreiras-pretas sem espinhos 'Arapaho', 'Xavante' e 'Ébano', com espinhos 'Comanche', 'Caingangue', 'Choctaw', 'Tupy', 'Guarani', 'Brazos' e 'Cherokee' e ainda da amoreira-vermelha, espécie nativa da serra da Mantiqueira do Brasil, foram produzidas por meio de estacas, segundo a metodologia de SILVA et al. (2012), e foram plantadas a campo em novembro de 2009, em espaçamento 3,0mx0,5m (densidade de 6.667 plantas por hectare). As plantas foram conduzidas sob espaldeira simples de um fio em " $T$ " (fios duplos paralelos), espaçados a $60 \mathrm{~cm}$ de distância e a $80 \mathrm{~cm}$ de altura do solo.

O experimento foi conduzido em blocos ao acaso, contendo 11 tratamentos (10 cultivares de amoreira-preta e uma espécie nativa), com quatro blocos e sete plantas úteis por unidade experimental.As plantas foram cultivadas seguindo as recomendações de CAMPAGNOLO \& PIO (2012c), para o cultivo de amoreiras em condições subtropicais. Durante a condução do experimento, foi realizado o controle das plantas espontâneas em toda a área experimental, além de duas aplicações de compostagem (3L por metro linear), uma em outubro e outra em dezembro, em cada ano de cultivo, sendo essa compostagem uma mistura decomposta de material vegetal e esterco de curral na proporção 3:1. Nos dois anos de cultivo, realizou-se análise do solo pelo método Mehlich 1. A análise revelou que o $\mathrm{pH}$ encontrava-se com o valor de 5,7 , além de $46,3 \mathrm{~g} \mathrm{dm}^{-3}$ de matéria orgânica, fósforo a $136,9 \mathrm{mg} \mathrm{dm}^{-3}$, cálcio a $10,1 \mathrm{mmolc} \mathrm{dm}^{-3}$, magnésio a 3,1 mmolc $\mathrm{dm}^{-3}$, soma de bases a 14,2 e CTC a 15,5 . Para manutenção das plantas, foram aplicados $200 \mathrm{~g}$ de sulfato de amônio, divididos em duas aplicações (uma em setembro e outra em janeiro), além de $100 \mathrm{~g}$ de superfosfato simples, $100 \mathrm{~g}$ de cloreto de potássio e $150 \mathrm{~g}$ de calcário por metro linear, aplicados em abril.

Nos ciclos produtivos 2010/11 e 2011/12, foram marcados os estádios fenológicos: início da brotação e início, término e duração da florada e da colheita.

As variáveis produtivas como número de frutos por planta, massa fresca dos frutos $(\mathrm{g})$, produção (g planta $\left.{ }^{-1}\right)$ e produtividade estimada $\left(\mathrm{kg} \mathrm{ha}^{-1}\right)$, foram avaliadas de abril a janeiro. Os frutos foram colhidos por parcela a cada três dias, contados e pesados. Ao final do ciclo de produção somaram-se todos os frutos e todas as massas registradas, para assim determinar a produção por planta e produtividade estimada. Foram coletadas as temperaturas médias máximas e mínimas e precipitação acumulada para os meses de março de 2010 a janeiro de 2012 (Figura 1).

Durante a plena colheita, no mês de dezembro, foram coletados 20 frutos por parcela, para a determinação das variáveis físicas (comprimento e diâmetro médio dos frutos) e variáveis químicas (acidez titulável, sólidos solúveis e a relação sólidos solúveis e acidez).

Ao final das análises de laboratório, os dados foram submetidos à análise de variância e as médias agrupadas pelo teste de Scott-Knott, a 5\% de probabilidade.

\section{RESULTADOS E DISCUSSÃO}

No primeiro ciclo produtivo (2010/11), todas as cultivares de amoreira-preta iniciaram a brotação no final da primeira quinzena do mês 


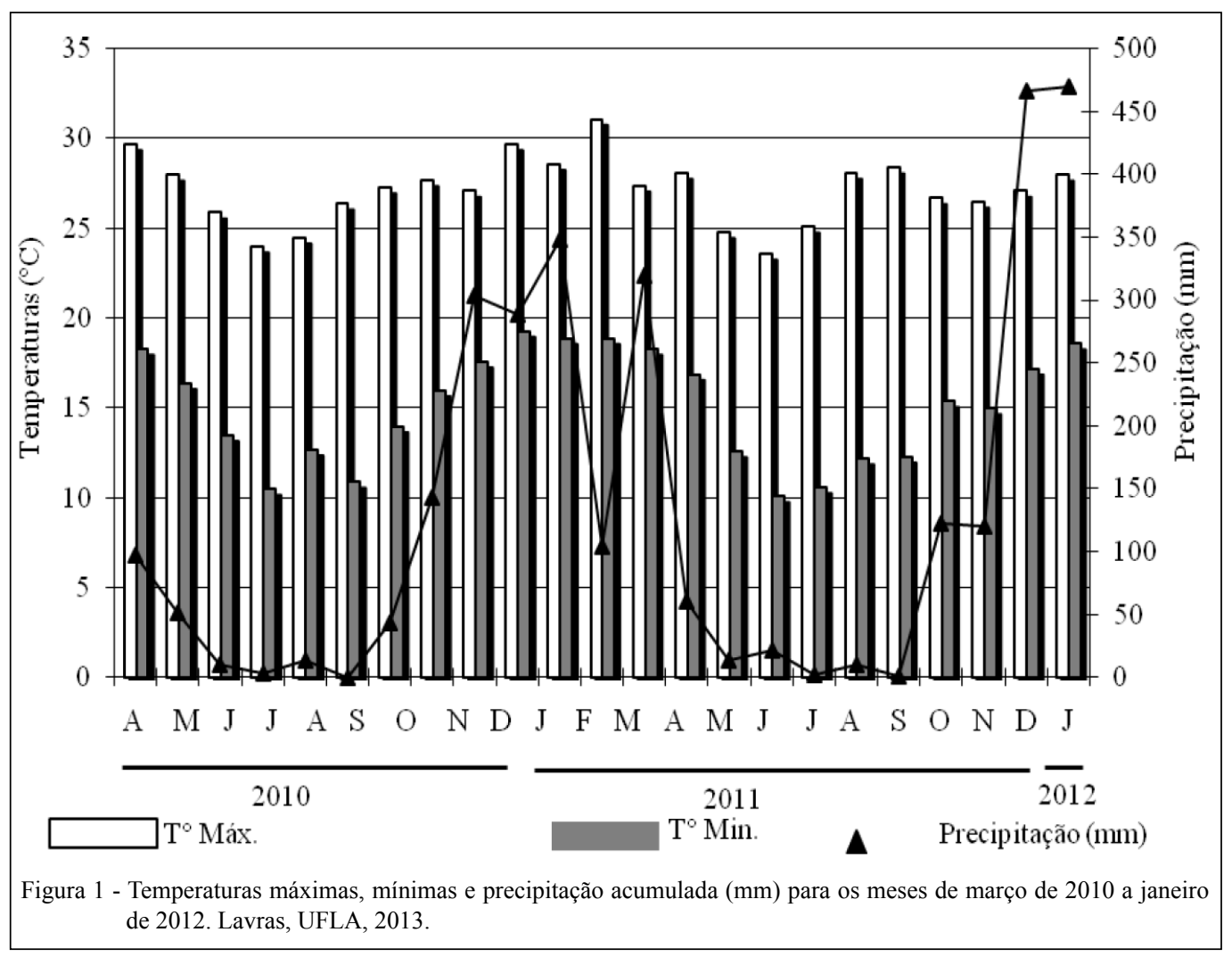

de julho (Tabela 1). Já no segundo ciclo, houve antecipação do início da brotação, possivelmente devido à maturidade das plantas ou devido ao clima, já que, no segundo ano, a média das temperaturas máximas de julho foi mais elevada (Figura 1). No caso da amoreira-vermelha, a brotação se iniciou no mês de março em ambos os ciclos produtivos.

Verificou-se que a floração das amoreiraspretas se iniciou em agosto no primeiro ciclo, a exceção das cultivares 'Caingangue' e 'Ébano', nas quais a emissão das flores se deu somente em setembro (Tabela 1). Já no segundo ciclo produtivo, houve antecipação do início da floração para a maioria das cultivares avaliadas, concentrando-se entre o final de julho e o final da primeira quinzena de agosto. Somente a cultivar 'Ébano' iniciou a emissão de flores no final de setembro. O término da floração no segundo ciclo foi mais prolongado, o que auxiliou no aumento do período da duração da floração das amoreiras-pretas, que persistiu entre 146 dias ('Tupy' e 'Brazos') e 162 dias ('Comanche'). Já nas plantas de amoreira-vermelha, a emissão das flores ocorreu em abril.

A duração da floração da amoreiravermelha foi a maior, e da amoreira-preta 'Ébano' a menor, em ambos os ciclos produtivos (Tabela 1). O mesmo resultado ocorreu com a duração da colheita. Além do mais, a 'Ébano' foi a mais tardia, pois as colheitas se iniciaram somente em novembro, ao passo que houve início da colheita dos frutos nas demais cultivares já no mês de setembro, sendo as cultivares mais precoces a 'Xavante', 'Arapaho' e 'Comanche'.

Fato surpreendente se deu na antecipação do período de colheita nas condições climáticas de Lavras-MG, em comparação à cidade de PelotasRS. Em Pelotas, a colheita da cultivar 'Brazos' se iniciou na segunda quinzena de novembro e se encerrou na primeira quinzena de janeiro, com duração média de 54 dias (ANTUNES et al., 2010). Em Lavras, a duração da colheita dessa mesma cultivar foi de 122 dias.

Este maior período de colheita é importante, pois verões quentes e interventos climáticos, como chuvas em demasia, concentradas no final do ano, como ocorrido no segundo ciclo produtivo (Figura 1), podem danificar os frutos e prejudicar as colheitas.

Com relação ao desempenho da produção, a maior produção de frutos no primeiro ciclo produtivo foi registrada com a amoreira-vermelha ( 715 frutos) e, posteriormente, com as cultivares de amoreirapreta 'Brazos', 'Comanche' e 'Guarani'. Apesar das 
Tabela 1 - Descrição fenológica dos ciclos produtivos 2010/11 e 2011/12 - início da brotação (IB), início, término e duração da floração (IF, TF e DF) e colheita (IC, TC e DC) de plantas de cultivares de amoreiras em Lavras - MG. Lavras, UFLA, 2013.

\begin{tabular}{|c|c|c|c|c|c|c|c|}
\hline Cultivares & $\mathrm{B}$ & IF & $\mathrm{TF}$ & $\mathrm{DF}(\text { dias })^{(1)}$ & $\mathrm{IC}$ & $\mathrm{TC}$ & $\mathrm{DC}(\operatorname{dias})^{(1)}$ \\
\hline \multicolumn{8}{|c|}{ 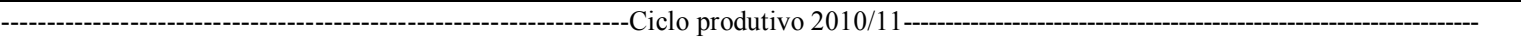 } \\
\hline Amora-vermelha & $13 / 03$ & $01 / 04$ & $13 / 12$ & 256 a & $14 / 04$ & $10 / 02$ & 302 a \\
\hline 'Xavante’ & $15 / 07$ & $10 / 08$ & $01 / 12$ & $113 \mathrm{~b}$ & $27 / 09$ & $26 / 01$ & $121 \mathrm{~b}$ \\
\hline ‘Tupy’ & $15 / 07$ & $17 / 08$ & $15 / 12$ & $120 \mathrm{~b}$ & $29 / 09$ & $26 / 01$ & $119 \mathrm{~b}$ \\
\hline 'Caingangue' & $15 / 07$ & 08/09 & $25 / 12$ & $108 \mathrm{~b}$ & $22 / 10$ & $28 / 01$ & $99 \mathrm{~b}$ \\
\hline 'Comanche' & $15 / 07$ & $11 / 08$ & $01 / 12$ & $112 \mathrm{~b}$ & $29 / 09$ & $31 / 01$ & $124 \mathrm{~b}$ \\
\hline 'Cherokee' & $15 / 07$ & $24 / 08$ & $05 / 12$ & $103 \mathrm{~b}$ & $11 / 10$ & $26 / 01$ & $107 \mathrm{~b}$ \\
\hline 'Brazos' & $15 / 07$ & $23 / 08$ & $01 / 12$ & $100 \mathrm{~b}$ & $01 / 10$ & $31 / 01$ & $122 \mathrm{~b}$ \\
\hline 'Arapaho' & $15 / 07$ & $13 / 08$ & $01 / 12$ & $115 \mathrm{~b}$ & $27 / 09$ & $26 / 01$ & $121 \mathrm{~b}$ \\
\hline 'Guarani’ & $15 / 07$ & $28 / 08$ & $10 / 12$ & $104 \mathrm{~b}$ & $01 / 10$ & $26 / 01$ & $117 \mathrm{~b}$ \\
\hline ‘Ébano’ & $15 / 07$ & $28 / 09$ & $10 / 12$ & $73 \mathrm{c}$ & $10 / 11$ & $31 / 01$ & $81 \mathrm{c}$ \\
\hline 'Choctaw' & $15 / 07$ & $15 / 08$ & $01 / 12$ & $108 \mathrm{~b}$ & $29 / 09$ & $26 / 01$ & $119 \mathrm{~b}$ \\
\hline C.V. $(\%)$ & - & - & - & 12,1 & - & - & 8,9 \\
\hline Amora-vermelha & $19 / 03$ & $06 / 04$ & $01 / 01$ & $270 \mathrm{a}$ & $19 / 04$ & $27 / 01$ & 283 a \\
\hline 'Xavante' & $07 / 07$ & $25 / 07$ & $01 / 01$ & $160 \mathrm{~b}$ & 03/09 & $11 / 01$ & $130 \mathrm{~b}$ \\
\hline ‘Tupy’ & $23 / 07$ & $15 / 08$ & 08/01 & $146 \mathrm{~b}$ & $23 / 09$ & $11 / 01$ & $110 \mathrm{~b}$ \\
\hline 'Caingangue' & 07/07 & 08/08 & $10 / 01$ & $155 \mathrm{~b}$ & $19 / 09$ & $11 / 01$ & $114 \mathrm{~b}$ \\
\hline 'Comanche' & 07/07 & $23 / 07$ & $01 / 01$ & $162 \mathrm{~b}$ & 05/09 & $16 / 01$ & $133 \mathrm{~b}$ \\
\hline 'Cherokee' & 07/07 & $05 / 08$ & $02 / 01$ & $150 \mathrm{~b}$ & $19 / 09$ & $11 / 01$ & $114 \mathrm{~b}$ \\
\hline 'Brazos' & $07 / 07$ & $08 / 08$ & $01 / 01$ & $146 \mathrm{~b}$ & $16 / 09$ & $16 / 01$ & $122 \mathrm{~b}$ \\
\hline 'Arapaho' & $07 / 07$ & $25 / 07$ & $01 / 01$ & $160 \mathrm{~b}$ & 08/09 & $11 / 01$ & $125 \mathrm{~b}$ \\
\hline 'Guarani' & $11 / 07$ & $10 / 08$ & $06 / 01$ & $151 \mathrm{~b}$ & $29 / 09$ & $11 / 01$ & $104 \mathrm{~b}$ \\
\hline ‘Ébano’ & $11 / 07$ & $26 / 09$ & $30 / 12$ & $95 \mathrm{c}$ & $01 / 11$ & $06 / 01$ & $66 \mathrm{c}$ \\
\hline 'Choctaw' & $07 / 07$ & $29 / 07$ & $01 / 01$ & $156 \mathrm{~b}$ & $12 / 09$ & $11 / 01$ & $121 \mathrm{~b}$ \\
\hline C.V. (\%) & & - & - & 11,3 & - & - & 8,1 \\
\hline
\end{tabular}

(1) Médias seguidas pela mesma letra não diferem entre si pelo teste Scott-Knott $(\mathrm{P}=0,05)$.

cultivares 'Tupy' e 'Guarani' terem produzido frutos de maior calibre, igualmente a 'Brazos', o número de frutos não foi suficiente para propiciar a produtividade de $12.379,1 \mathrm{~kg} \mathrm{ha}^{-1}$, registrada para a 'Brazos'. No entanto, essas duas cultivares proporcionaram boa produtividade, registrando $9.031,4 \mathrm{~kg} \mathrm{ha}^{-1} \mathrm{e} 7.593,1 \mathrm{~kg}$ $\mathrm{ha}^{-1}$, respectivamente (Tabela 2). Já no caso da amoreira-vermelha, devido ao tamanho diminuto de seus frutos $(1,3 \mathrm{~g})$, a produtividade foi baixa $\left(3.514,0 \mathrm{~kg} \mathrm{ha}^{-1}\right)$.

Já no segundo ciclo produtivo, novamente, a amoreira-vermelha e as cultivares 'Brazos', 'Comanche' e 'Guarani' produziram maior número de frutos, juntamente com a 'Arapaho'. Porém, somente 'Brazos' produziu frutos de maior calibre $(10,1 \mathrm{~g})$ e, consequentemente, obteve a maior produção por planta $(3 \mathrm{~kg})$, atingindo assim a produtividade estimada de $20.032,1 \mathrm{~kg} \mathrm{ha}^{-1}$, incremento de $7.653,0 \mathrm{~kg}$ $\mathrm{ha}^{-1}$ em relação ao ciclo produtivo anterior.

Os resultados encontrados neste trabalho foram superiores aos obtidos por ANTUNES et al.
(2010), que avaliaram o desempenho produtivo de cultivares de amoreira-preta nas condições de clima temperado de Pelotas-RS (clima Cfa) e registraram produtividade média de $2.794 \mathrm{~kg} \mathrm{ha}^{-1}$ com a cultivar 'Brazos', $17.238,1 \mathrm{~kg} \mathrm{ha}^{-1}$ a menos em relação aos resultados obtidos em Lavras-MG. Por outro lado, o resultado com a cultivar 'Brazos' foi próximo ao registrado por ANTUNES et al. (2000) em CaldasMG (região de clima subtropical).

No entanto, RASEIRA et al. (2007) registraram a produtividade de $15.200 \mathrm{~kg} \mathrm{ha}^{-1} \mathrm{com}$ a cultivar de amoreira-preta 'Ébano' em Pelotas-RS. No presente trabalho, foram obtidos apenas $5.074,8 \mathrm{~kg}$ $\mathrm{ha}^{-1}$ no primeiro ciclo de avaliação e $4.431,9 \mathrm{~kg} \mathrm{ha}^{-1}$ no segundo ciclo (Tabela 2). O mesmo resultado ocorreu com as cultivares 'Arapaho' e 'Choctaw', pois foram obtidas as produtividades de $3.130,5 \mathrm{~kg}$ $\mathrm{ha}^{-1}$ e $6.646,9 \mathrm{~kg} \mathrm{ha}^{-1}$, respectivamente, no segundo ciclo de avaliação (Tabela 2). Esses resultados são inferiores ao obtido por CLARK et al. (2005), que registraram produtividades de $7.365 \mathrm{~kg} \mathrm{ha}^{-1}$ com a 
Tabela 2 - Número médio de frutos, massa fresca média, produção e produtividade estimada dos ciclos produtivos 2010/11 e 2011/12 de plantas de cultivares de amoreiras em Lavras - MG. Lavras, UFLA, 2013.

\begin{tabular}{|c|c|c|c|c|}
\hline Cultivares & $\mathrm{N}^{\mathrm{o}}$ frutos & Massa fresca $(\mathrm{g})$ & Produção por planta $(\mathrm{g})$ & $\begin{array}{l}\text { Produtividade estimada } \\
\qquad\left(\mathrm{kg} \mathrm{ha}^{-1}\right)^{(2)}\end{array}$ \\
\hline \multicolumn{5}{|c|}{ 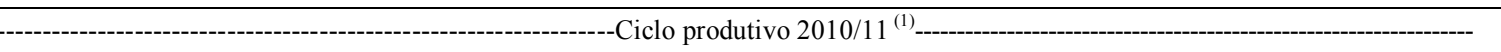 } \\
\hline Amora-vermelha & 715,3 a & $1,3 \mathrm{~d}$ & $527,3 \mathrm{~d}$ & $3.514,6 \mathrm{~d}$ \\
\hline 'Xavante' & $67,9 \mathrm{~d}$ & $5,4 \mathrm{c}$ & $218,8 \mathrm{f}$ & $1.459,1 \mathrm{f}$ \\
\hline ‘Tupy’ & $205,5 \mathrm{c}$ & 8,6 a & $1.354,6 \mathrm{~b}$ & $9.031,4 \mathrm{~b}$ \\
\hline 'Caingangue' & $46,2 \mathrm{~d}$ & $6,1 \mathrm{~b}$ & $214,1 \mathrm{f}$ & $1.432,1 \mathrm{f}$ \\
\hline 'Comanche' & $277,0 \mathrm{~b}$ & $5,4 \mathrm{c}$ & $982,4 \mathrm{~b}$ & $6.549,9 \mathrm{c}$ \\
\hline 'Cherokee' & $97,6 \mathrm{~d}$ & $5,1 \mathrm{c}$ & $336,1 \mathrm{e}$ & $2.240,9 \mathrm{e}$ \\
\hline 'Brazos' & $261,4 \mathrm{~b}$ & 8,2 a & $1.856,8 \mathrm{a}$ & $12.379,1 \mathrm{a}$ \\
\hline 'Arapaho' & $88,2 \mathrm{~d}$ & $5,6 \mathrm{c}$ & $324,3 \mathrm{e}$ & $2.162,1 \mathrm{e}$ \\
\hline 'Guaran'i & $234,2 \mathrm{~b}$ & 8,7 a & $1.138,9 \mathrm{~b}$ & $7.593,1 \mathrm{~b}$ \\
\hline 'Ébano’ & $198,5 \mathrm{c}$ & $5,9 \mathrm{~b}$ & $761,2 \mathrm{c}$ & $5.074,8 \mathrm{c}$ \\
\hline 'Choctaw' & $209,3 \mathrm{c}$ & $6,5 \mathrm{~b}$ & $1.003,6 \mathrm{~b}$ & $6.691,3 \mathrm{c}$ \\
\hline C.V. $(\%)$ & 8,5 & 8,7 & 11,1 & 11,1 \\
\hline Amora-vermelha & 330,7 a & $\begin{array}{l}\text { produtivo } 2011 / \\
2,7 \mathrm{e}\end{array}$ & $344,1 \mathrm{~d}$ & $2.294,1 \mathrm{f}$ \\
\hline 'Xavante' & $106,6 \mathrm{c}$ & $4,7 \mathrm{~d}$ & $450,8 \mathrm{~d}$ & $3.005,7 \mathrm{f}$ \\
\hline ‘Tupy’ & $259,2 \mathrm{~b}$ & $6,8 \mathrm{c}$ & $2.074,8 \mathrm{~b}$ & $13.832,7 \mathrm{c}$ \\
\hline 'Caingangue' & $216,8 \mathrm{~b}$ & $5,1 \mathrm{~d}$ & $956,6 \mathrm{c}$ & $6.377,5 \mathrm{e}$ \\
\hline 'Comanche' & $345,5 \mathrm{a}$ & $5,6 \mathrm{c}$ & $1.463,7 \mathrm{c}$ & $9.758,6 \mathrm{~d}$ \\
\hline 'Cherokee' & $168,8 \mathrm{c}$ & $4,5 \mathrm{~d}$ & $671,2 \mathrm{~d}$ & $4.474,9 \mathrm{f}$ \\
\hline 'Brazos' & $403,2 \mathrm{a}$ & $10,1 \mathrm{a}$ & $3.004,6 \mathrm{a}$ & $20.032,1 \mathrm{a}$ \\
\hline ‘Arapaho’ & 320,7 a & $4,6 \mathrm{~d}$ & $465,8 \mathrm{~d}$ & $3.130,5 \mathrm{f}$ \\
\hline 'Guarani' & 376,7 a & $8,5 \mathrm{~b}$ & $2.539,0 \mathrm{~b}$ & $16.927,5 \mathrm{~b}$ \\
\hline ‘Ébano’ & $130,7 \mathrm{c}$ & $5,6 \mathrm{c}$ & $664,7 \mathrm{~d}$ & $4.431,9 \mathrm{f}$ \\
\hline 'Choctaw' & $212,8 \mathrm{~b}$ & $6,8 \mathrm{c}$ & $996,9 \mathrm{c}$ & $6.646,9 \mathrm{e}$ \\
\hline $\mathrm{CV}(\%)$ & 16,5 & 12,7 & 14,3 & 14,3 \\
\hline
\end{tabular}

${ }^{(1)}$ Médias seguidas pela mesma letra não diferem entre si pelo teste Scott-Knott $(\mathrm{P}=0,05)$.

${ }^{\text {(2) }}$ Cálculo considerando espaçamento $3,0 \mathrm{~m} \times 0,5 \mathrm{~m}$, com densidade de 6.667 plantas por hectare.

cultivar 'Arapaho' e 16.891 $\mathrm{kg} \mathrm{ha}^{-1}$ com a 'Choctaw', em trabalho realizado em Hope city, estado de

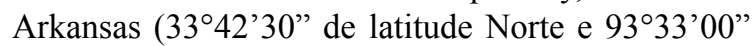
de latitude Oeste, local com temperaturas negativas nos meses mais frios).

Para a qualidade dos frutos produzidos, as maiores dimensões foram registradas para as cultivares 'Brazos', 'Guarani' e 'Tupy' no primeiro ciclo de avaliação e somente para a 'Brazos' no segundo ciclo (Tabela 3). Ressalta-se que, no primeiro ciclo, a cultivar 'Ébano' registrou maior diâmetro de frutos, mas, como seus frutos possuem formato arredondado, o comprimento foi inferior em comparação às demais cultivares.

Frutos das cultivares 'Tupy' e 'Caingangue' apresentaram menor acidez no primeiro ciclo e novamente a 'Caingangue' no segundo ciclo, enquanto os frutos das cultivares 'Xavante' e 'Ébano' e ainda a amora-vermelha foram mais ácidos (Tabela 3).
Quanto aos teores de sólidos solúveis, frutos da amoreira-vermelha e das cultivares 'Caingangue' e 'Cherokee' registraram maiores índices no primeiro ciclo e novamente a 'Caingangue' no segundo ciclo de avaliação (Tabela 3). Mas, devido à acidez dos frutos, a relação mais alta entre a concentração de sólidos solúveis e acidez foi para a cultivar 'Caingangue', seguida da 'Tupy' nos dois ciclos de avaliação, incluindo a 'Choctaw' no segundo ciclo (Tabela 3), sendo essas indicadas para o consumo como frutas frescas.

Como o desempenho produtivo da cultivar 'Tupy' foi superior, com $13.832,7 \mathrm{~kg} \mathrm{ha}^{-1}, 50 \%$ a mais em relação as cultivares 'Caingangue' $(6.377,5 \mathrm{~kg}$ ha $^{-1}$ ) e 'Choctaw' (6.646,9 $\mathrm{kg} \mathrm{ha}^{-1}$ ) (Tabela 2), a opção para a produção de frutos visando ao mercado de fruta fresca seria a utilização da 'Tupy' e, para a produção de frutos destinados ao processamento, a 'Brazos'. Outra opção visando à produção de doces 
Tabela 3 - Comprimento, diâmetro, acidez titulável, sólidos solúveis (SS, expresso em ${ }^{\circ}$ Brix) e relação SST/acidez em frutos oriundos dos ciclos produtivos 2010/11 e 2011/12 de plantas de cultivares de amoreiras em Lavras - MG. Lavras, UFLA, 2013.

\begin{tabular}{|c|c|c|c|c|c|}
\hline Cultivares & Comprimento $(\mathrm{mm})^{(1)}$ & Diâmetro (mm) & Acidez (g/100g) & SS $\left({ }^{\circ}\right.$ Brix $)$ & Relação SS/Acidez \\
\hline \multicolumn{6}{|c|}{ 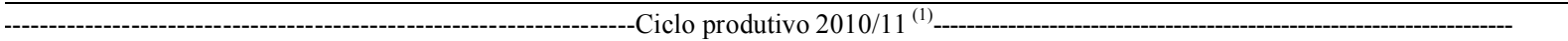 } \\
\hline Amora-vermelha & $16,5 \mathrm{~d}$ & $17,3 \mathrm{~d}$ & $1,1 \mathrm{c}$ & 11,1 a & $10,1 \mathrm{~b}$ \\
\hline 'Xavante' & $23,2 \mathrm{c}$ & $20,9 \mathrm{c}$ & $1,1 \mathrm{c}$ & $9,2 \mathrm{c}$ & $8,4 \mathrm{c}$ \\
\hline ‘Tupy’ & 27,7 a & 24,0 a & $0,9 \mathrm{~d}$ & $8,7 \mathrm{~d}$ & $9,7 \mathrm{~b}$ \\
\hline 'Caingangue' & $23,1 \mathrm{c}$ & $22,5 \mathrm{~b}$ & $0,8 \mathrm{~d}$ & $9,6 \mathrm{~b}$ & $12,0 \mathrm{a}$ \\
\hline 'Comanche' & $22,3 \mathrm{c}$ & $21,0 \mathrm{c}$ & $1,3 \mathrm{~b}$ & $8,1 \mathrm{e}$ & $6,2 \mathrm{~d}$ \\
\hline 'Cherokee' & $21,9 \mathrm{c}$ & $19,1 \mathrm{~d}$ & $1,2 \mathrm{c}$ & $9,6 \mathrm{~b}$ & $8,0 \mathrm{c}$ \\
\hline 'Brazos’' & $26,5 \mathrm{a}$ & 24,1 a & $1,1 \mathrm{c}$ & $7,4 \mathrm{f}$ & $6,7 \mathrm{c}$ \\
\hline 'Arapaho' & $22,9 \mathrm{c}$ & $21,0 \mathrm{c}$ & $1,1 \mathrm{c}$ & $8,3 \mathrm{e}$ & $7,5 \mathrm{c}$ \\
\hline 'Guarani' & 28,1 a & 23,8 a & $1,1 \mathrm{c}$ & $7,8 \mathrm{f}$ & $7,1 \mathrm{c}$ \\
\hline ‘Ébano' & $22,9 \mathrm{c}$ & 23,4 a & $1,6 \mathrm{a}$ & $8,4 \mathrm{e}$ & $5,2 \mathrm{~d}$ \\
\hline 'Choctaw' & $24,8 \mathrm{~b}$ & $22,5 \mathrm{~b}$ & $1,1 \mathrm{c}$ & $9,0 \mathrm{c}$ & $8,1 \mathrm{c}$ \\
\hline $\mathrm{CV}(\%)$ & 5,1 & 4,1 & 13,8 & 2,8 & 3,4 \\
\hline \multicolumn{6}{|c|}{--Ciclo produtivo $2011 / 12^{(1)}$} \\
\hline Amora-vermelha & $16,6 \mathrm{~d}$ & $22,9 \mathrm{c}$ & $1,6 \mathrm{a}$ & $9,7 \mathrm{~b}$ & $6,1 \mathrm{~d}$ \\
\hline 'Xavante' & $24,1 \mathrm{c}$ & $21,8 \mathrm{c}$ & 1,6 a & $8,0 \mathrm{c}$ & $5,0 \mathrm{~d}$ \\
\hline ‘Tupy’ & $28,5 \mathrm{~b}$ & $22,4 \mathrm{c}$ & $0,9 \mathrm{c}$ & $8,4 \mathrm{c}$ & $9,3 \mathrm{~b}$ \\
\hline 'Caingangue' & $22,5 \mathrm{c}$ & $21,3 \mathrm{c}$ & $0,7 \mathrm{~d}$ & $11,2 \mathrm{a}$ & $16,0 \mathrm{a}$ \\
\hline 'Comanche' & $25,6 \mathrm{c}$ & $21,7 \mathrm{c}$ & $1,3 \mathrm{~b}$ & $8,9 \mathrm{c}$ & $6,8 \mathrm{c}$ \\
\hline 'Cherokee' & $23,4 \mathrm{c}$ & $20,4 \mathrm{c}$ & $1,0 \mathrm{c}$ & $8,2 \mathrm{c}$ & $8,2 \mathrm{c}$ \\
\hline 'Brazos' & 32,0 a & 27,2 a & $1,2 \mathrm{~b}$ & $8,5 \mathrm{c}$ & $7,1 \mathrm{c}$ \\
\hline 'Arapaho' & $22,8 \mathrm{c}$ & $21,2 \mathrm{c}$ & $1,1 \mathrm{c}$ & $8,4 \mathrm{c}$ & $7,6 \mathrm{c}$ \\
\hline 'Guarani' & $29,3 \mathrm{~b}$ & $24,9 \mathrm{~b}$ & $1,2 \mathrm{~b}$ & $8,2 \mathrm{c}$ & $6,8 \mathrm{c}$ \\
\hline ‘Ébano’ & $25,0 \mathrm{c}$ & $23,4 \mathrm{c}$ & $1,6 \mathrm{a}$ & $6,9 \mathrm{~d}$ & $4,3 \mathrm{~d}$ \\
\hline 'Choctaw' & $27,2 \mathrm{~b}$ & $24,6 \mathrm{~b}$ & $1,0 \mathrm{c}$ & $9,5 \mathrm{~b}$ & $9,5 \mathrm{~b}$ \\
\hline CV. $(\%)$ & 6,3 & 6,7 & 16,5 & 7,9 & 4,4 \\
\hline
\end{tabular}

(1) Médias seguidas pela mesma letra não diferem entre si pelo teste $\operatorname{Scott}-\operatorname{Knott}(\mathrm{P}=0,05)$.

seria a utilização dos frutos da amoreira-vermelha, devido ao seu tamanho diminuto, alta acidez e alto teor de sólidos solúveis.

Ressalta-se que podem ainda ocorrer variações nos teores dos compostos químicos em detrimento do local onde são cultivadas, em razão das diferenças quanto à intensidade de radiação solar e amplitude térmica, que influenciam nas características organolépticas dos frutos (ALI et al., 2011). Isso é comprovado pelos resultados obtidos por ANTUNES et al. (2010), que registraram $8,9^{\circ}$ Brix para a cultivar 'Caingangue', sendo $2,3^{\circ}$ Brix a menos em comparação aos obtidos com essa cultivar no presente trabalho.

\section{CONCLUSÃO}

A maioria das cultivares apresenta ciclo produtivo superior a 100 dias. 'Brazos', 'Guarani' e 'Tupy' produzem frutos de maior massa, porém a 'Brazos' obteve a maior produtividade estimada, sendo a mais promissora para a região de Lavras-MG. 'Cainguangue', 'Tupy'e ‘Choctaw' apresentam o melhor equilíbrio entre os sólidos solúveis e a acidez. Aamoreiravermelha apresenta boa produção e demonstrou-se uma excelente opção para o processamento.

\section{AGRADECIMENTOS}

Os autores agradecem ao Conselho Nacional de Desenvolvimento Científico e Tecnológico (CNPq), a Coordenação de Aperfeiçoamento de Pessoal de Nivel Superior (CAPES) e a Fundação de Amparo à Pesquisa do Estado de Minas Gerais (FAPEMIG) pelo auxílio financeiro.

\section{REFERÊNCIAS}

ALI, L. et al. Late season harvest and storage of Rubus berries: major antioxidant and sugar levels. Scientia Horticulturae, v.129, n.3, p.376-381, 2011. Disponível em: <http://www.sciencedirect. com/science/article/pii/S0304423811001701>. Acesso em: 20 jan. 2015. doi: 10.1016/j.scienta.2011.03.047.

ANTUNES, L.E.C. et al. Fenologia e produção de cultivares de amoreira-preta em sistema agroecológico. Ciência Rural, v.40, 
n.9, p.1929-1933, 2010. Disponível em: <http://www.scielo. br/scielo.php?pid $=$ S0103-84782010000900012\&script $=$ sci arttext>. Acesso em: 24 out. 2013. doi: 10.1590/S010384782010000900012

ANTUNES, L.E.C. et al. Fenologia e produção de variedades de amora-preta nas condições do planalto de Poços de Caldas-MG. Revista Brasileira de Fruticultura, v.22, n.1, p.89-95, 2000.

CAMPAGNOLO, M.A.; PIO, R. Produção da amoreira-preta 'Tupy' sob diferentes épocas de poda. Ciência Rural, v.42, n.2, p.225-231, 2012a. Disponível em: <http://www.scielo. $\mathrm{br} / \mathrm{scielo} . \mathrm{php}$ ? pid $=\mathrm{S} 0103-84782012000200007 \& \mathrm{script}=\mathrm{sci}$ arttext $>$. Acesso em: 12 nov. 2013. doi: 10.1590/S010384782012005000007

CAMPAGNOLO, M.A.; PIO, R. Phenological and yield performance of black and redberry cultivars in western Paraná State. Acta Scientiarum. Agronomy, v.34, n.4, p.439-444, 2012b. Disponível em: <http://www.scielo.br/scielo.php?pid=S180786212012000400011\&script $=$ sci_arttext $>$. Acesso em: 14 nov. 2013. doi: 10.4025/actasciagron.v34i4.15528.

CAMPAGNOLO, M.A.; PIO, R. Poda drástica para a produção da amora-preta em regiões subtropicais. Pesquisa Agropecuária Brasileira, v.47, n.7, p.934-938, 2012c. Disponível em: <http://www.scielo.br/scielo.php?pid=S0100204X2012000700009\&script $=$ sci_arttext $>$. Acesso em: 17 nov. 2013. doi: 10.1590/S0100-204X2012000700009.

CLARK, J.R. et al. 'Prime-Jan' ('APF 8') and 'Prime-Jim' (APF 12') primocane fruiting blackberry. Hortscience, v. 40 p.852-855, 2005. Disponível em: <http://arsserv0.tamu.edu/ SP2UserFiles/person/1718/PDF/2005/Prime-Jan2005.pdf >. Acesso em: 11 nov. 2013.

CURI, P.N. et al. Qualidade de framboesas sem cobertura ou cobertas sobre o dossel e em diferentes espaçamentos. Revista Brasileira de Fruticultura, Jaboticabal, v.36, n.1, p.199-205, 2014. Disponível em: <http://www.scielo.br/ scielo.php?script $=$ sci_arttext\&pid $=\mathrm{S} 01002945201400010002$ $3 \& \operatorname{lng}=$ pt\&nrm=iso\&tlng=en $>$. Acesso em: 19 set. 2014. doi: $10.1590 / 0100-2945-234 / 13$.
GUEDES, M.N.S. et al. Composição química, compostos bioativos e dissimilaridade genética entre cultivares de amoreira (Rubus spp.) cultivadas no Sul de Minas Gerais. Revista Brasileira de Fruticultura, Jaboticabal, v.36, n.1, p.206-213, 2014. Disponível em: $<$ http://www.scielo.br/scielo.php?script=sci arttext\&pid=S0100-29452014000100024\&lng=pt\&nrm=iso\&tlng $=$ en $>$. Acesso em: 19 set. 2014. doi: 10.1590/0100-2945-230/13.

GUEDES, M.N.S. et al. Chemical characterization and mineral levels in the fruits of blackberry cultivars grown in a tropical climate at an elevation. Acta Scientiarum. Agronomy, Maringá, v.32, n.2, p.191-196, 2013. Disponível em: <http://www.scielo.br/ scielo.php?pid $=$ S1807-86212013000200007\&script $=$ sci_arttext $>$. Acesso em: 05 nov. 2013. doi: 10.4025/actasciagron.v35i2.16630.

MARO, L.A.C. et al. Environmental and genetic variation in the post-harvest quality of raspberries in subtropical areas in Brazil. Acta Scientiarum. Agronomy, Maringá, v.36, n.3, p.323-328, 2014. Disponível em: <http://periodicos.uem.br/ojs/index.php/ ActaSciAgron/article/view/18050>. Acesso em: 19 set. 2014. doi: 10.4025/actasciagron.v36i3.18050.

RASEIRA, A. et al. Influência da densidade de plantio na produtividade de cultivares de amoreira-preta. Revista Brasileira de Agrociência, v.13, n.4, p.551-554, 2007. Disponível em: $<$ http://www.ufpel.edu.br/faem/agrociencia/v13n4/artigo21.pdf $>$. Acesso em: 13 nov. 2013

SILVA, K.N. et al. Produção de mudas de framboeseira negra por diferentes métodos de propagação vegetativa. Ciência Rural, Santa Maria, v.42, n.3, p.418-422, 2012. Disponível em: $<$ http://www.scielo.br/scielo.php?pid=S0103$84782012000300006 \&$ script $=$ sci arttext $>$. Acesso em: 23 out. 2013. doi: 10.1590/S0103-84782012000300006.

SOUZA, V.R. et al. Determination of the bioactive compounds, antioxidant activity and chemical composition of Brazilian blackberry, red raspberry, strawberry, blueberry and sweet cherry fruits. Food Chemistry, v.156, p.362-368, 2014. Disponível em: $<$ http://ac.els-cdn.com/S0308814614001770/1s2.0S03088146140 01770main.pdf? tid=f1425554bbf411e3aa8900000aab0f6b\&acdn at $=1396614824 \_0 d a 62$ cdedaf5c9f85864d781725edb24>. Acesso em: 02 abr. 2014. doi: 10.1016/j.foodchem.2014.01.125. 